\title{
Location and Size of Dopaminergic and Serotonergic Cell Populations Are Controlled by the Position of the Midbrain- Hindbrain Organizer
}

\author{
Claude Brodski, ${ }^{1 *}$ Daniela M. Vogt Weisenhorn, ${ }^{1 *}$ Massimo Signore, ${ }^{2 *}$ Inge Sillaber, ${ }^{1}$ Matthias Oesterheld, ${ }^{1}$ \\ Vania Broccoli, ${ }^{3}$ Dario Acampora, ${ }^{2}$ Antonio Simeone, ${ }^{2}$ and Wolfgang Wurst ${ }^{1}$ \\ ${ }^{1}$ Max-Planck-Institute of Psychiatry, 80804 Munich, Germany, and GSF-National Research Center for Environment and Health, Technical University \\ Munich, Institute of Developmental Genetics, 85758 Oberschleissheim, Germany, ${ }^{2}$ Medical Research Council Centre for Developmental Neurobiology, \\ King's College London, London SE1 UL, United Kingdom, and Institute of Genetics and Biophysics, Consiglio Nazionale delle Ricerche, 80125 Naples, Italy, \\ and ${ }^{3}$ Stem Cell Research Institute, Department of Biological and Technological Research-San Raffaele Hospital, I-20132 Milan, Italy
}

\begin{abstract}
Midbrain dopaminergic and hindbrain serotonergic neurons play an important role in the modulation of behavior and are involved in a series of neuropsychiatric disorders. Despite the importance of these cells, little is known about the molecular mechanisms governing their development. During embryogenesis, midbrain dopaminergic neurons are specified rostral to the midbrain-hindbrain organizer (MHO), and hindbrain serotonergic neurons are specified caudal to it. We report that in transgenic mice in which $0 t x 2$ and accordingly the MHO are shifted caudally, the midbrain dopaminergic neuronal population expands to the ectopically positioned MHO and is enlarged. Complementary, the extension of the hindbrain serotonergic cell group is decreased. These changes are preserved in adulthood, and the additional, ectopic dopaminergic neurons project to the striatum, which is a proper dopaminergic target area. In addition, in mutants in which Otx2 and the MHO are shifted rostrally, dopaminergic and serotonergic neurons are relocated at the newly positioned MHO. However, in these mice, the size ratio between these two cell populations is changed in favor of the serotonergic cell population. To investigate whether the position of the MHO during embryogenesis is also of functional relevance for adult behavior, we tested mice with a caudally shifted MHO and report that these mutants show a higher locomotor activity. Together, we provide evidence that the position of the MHO determines the location and size of midbrain dopaminergic and hindbrain serotonergic cell populations in vivo. In addition, our data suggest that the position of the MHO during embryogenesis can modulate adult locomotor activity.
\end{abstract}

Key words: development; substantia nigra; ventral tegmental area; raphe nuclei; isthmic organizer; hyperactivity

\section{Introduction}

Midbrain dopaminergic (mid-DA) neurons of the substantia nigra (SN), ventral tegmental area (VTA), and retrorubral field $(\mathrm{RrF})$ project to the striatum, cortex, and nucleus accumbens (Björklund and Lindvall, 1984). They modulate a variety of functions, such as movement, rewarding, cognition, and feeding (Zhou and Palmiter, 1995; Cooper et al., 2001). A dysfunction of these cells is responsible for Parkinson's disease and is thought to be involved in schizophrenia and attention-deficit hyperactivity disorder (ADHD) (Waldman et al., 1998; Barr et al., 2001; Cooper et al., 2001). Hindbrain serotonergic (5-HT) neurons are grouped in a rostral cluster (rost-5-HT) projecting to the mid-

\footnotetext{
Received Aug. 8, 2002; revised Feb. 12, 2003; accepted Feb. 18, 2003.

This work was supported by Medical Research Council Grant G9900955 (A.S.), the Wellcome Trust 062642/Z/00 (A.S.), the Ministero dell'Università a della Ricerca Scientifica e Tecnologica-Consiglio Nazionale delle Ricerche Programme Legge 488/92 (cluster 02) (A.S.), the Bettencourt Schueller Foundation (A.S.), the Bundesministerium für Bildung und Forschung (W.W.), and the Deutsche Forschungsgemeinschaft (W.W.). We thank L. Bally-Cuif, G. Dechant, and N. Prakash for critically reading this manuscript and for valuable discussions; A. M. Lucchese, S. Pirrung, L. Sonnier, U. Genning, and Ch. Bartl for excellent technical support; G. Martin for Fgf8; A. von Holst for Pet1 probe; and J. Preil for expert help applying the method of unbiased stereology.

*C.B., D.M.V.W., and M.S. contributed equally to this work.

Correspondence should be addressed to either of the following: Wolfgang Wurst, Max-Planck-Institute of Psychiatry, Kraepelinstrasse 2-10, 80804 Munich, Germany, E-mail: wurst@gsf.de; or Antonio Simeone, Medical Research Council Centre for Developmental Neurobiology, King's College London, Guy's Campus, New Hunts House, London SE1 1UL, UK, E-mail: antonio.simeone@kcl.ac.uk.

Copyright (C) 2003 Society for Neuroscience $\quad$ 0270-6474/03/234199-09\$15.00/0
}

brain and forebrain and a caudal cluster innervating the spinal cord (Tork, 1990). The rost-5-HT cells are implicated in a wide range of processes, such as mood, vegetative homeostasis (Cooper et al., 2001), and movement control (Jacobs and Fornal, 1997). A dysregulation of these neurons is supposed to be associated with affective diseases and attention-deficit hyperactivity disorder (Quist et al., 2000; Cooper et al., 2001).

The molecular mechanisms directing the development of mid-DA and rost-5-HT neurons in vivo are still poorly understood (Goridis and Rohrer, 2002). The genetic inactivation of the orphan nuclear receptor Nurr1, which is widely expressed in the brain, leads to failure in the maturation of mid-DA neurons (Zetterström et al., 1997). Other transcription factors, such as $P t \times 3$, which is also known as Pitx3, and $L m \times 1 b$, are expressed almost exclusively in mid-DA neurons in the brain, but they do not seem to be required for the induction of neurotransmitter identity (Smidt et al., 1997, 2000). Experiments using an explant culture system have demonstrated that the secreted factors fibroblast growth factor 8 (FGF8) and sonic hedgehog ( $\mathrm{SHH}$ ) are required for the induction of mid-DA neurons rostral to the midbrain-hindbrain organizer (MHO) and rost-5-HT cells caudal to it (Ye et al., 1998). Although these studies identified several genes necessary for the development of mid-DA and rost-5-HT neurons, it is still unclear how the location and size of these cell populations are determined in vivo. 
During embryogenesis, the boundary between the midbrain and hindbrain acts as an organizing center $(\mathrm{MHO})$, directing the patterning of the adjacent territories (Martinez and AlvaradoMallart, 1990; Martinez et al., 1991; Marin and Puelles, 1994; Wassef and Joyner, 1997; Wurst and Bally-Cuif, 2001). At the onset of somitogenesis, the transcription factor Otx2 is expressed directly rostral to the $\mathrm{MHO}$ in the midbrain and forebrain, whereas the transcription factor $G b \times 2$ is expressed directly caudal to it in the hindbrain and spinal cord. Shifting the expression domain of either gene (Broccoli et al., 1999; Millet et al., 1999) or reducing the level of OTX proteins below a critical threshold (Acampora et al., 1997; Suda et al., 1997) leads to a caudal or rostral shift of the organizer, indicating that the expression border between Otx2 and Gbx2 defines the position of the MHO (Hidalgo-Sanchez et al., 1999; Katahira et al., 2000; Simeone, 2000; Garda et al., 2001; Li and Joyner, 2001; Martinez-Barbera et al., 2001; Wurst and Bally-Cuif, 2001). Furthermore, a number of other genes expressed at the MHO, such as Fgf8, En1, En2, Wnt1, Pax2, and Pax5, are involved in the maintenance of this territory (McMahon et al., 1992; Millen et al., 1994; Wurst et al., 1994; Favor et al., 1996; Schwarz et al., 1997; Meyers et al., 1998).

Using two transgenic mouse models in which the MHO is shifted either caudally or rostrally, we provide evidence that the position of the MHO determines the location and the size of the mid-DA and rost-5-HT neuronal populations in vivo. In addition, our data suggest that the position of the MHO during embryogenesis is of functional relevance for adult locomotor activity.

\section{Materials and Methods}

Transgenic mice. The generation of $E n 1^{+/ O t x 2}$ mutants has been described previously (Broccoli et al., 1999). They are bred in a mixed C57BL/6, CD-1, and 129/SV genetic background.

$O t x 1^{-/-} ; O t x 2^{+/-}$mice were generated by crossing $O t x 1^{+/-} ; O t x 2^{+/-}$ males with $\mathrm{Otx} 1^{+/-} ; \mathrm{Otx} 2^{+/+}$females. The offspring were obtained by brother-sister breedings in a C57BL/6, 129/SV, DBA2 genetic background.

Gene expression studies. Brains of adult and embryonic mice were either transcardially perfused or immersion fixed with $4 \%$ paraformaldehyde (PFA). Some of the adult brains were shock frozen on dry ice. Perfused brains were either cut on a cryostat in 30 - to $50-\mu \mathrm{m}$-thick sections or paraffin embedded and cut on a microtome in 8 - $\mu$ m-thick sections. Frozen brains were cut on a cryostat in $18-\mu$ m-thick sections. All sections were processed for in situ hybridization according to a modified version of the procedure described by Dagerlind et al. (1992). Antisense and sense mRNA probes were transcribed from plasmids containing fragments of the murine tyrosine hydroxylase $(T h)$ gene (base pairs 1-760; GenBank accession number M69200), the murine serotonin-transporter gene (base pairs 1967-2481; GenBank accession number AF013604), the murine Pet-1 gene (base pairs 905-1454; GenBank accession number AY049085), the murine dopamine-transporter gene (base pairs 2333-2724; GenBank accession number AF109391), the murine Ptx3 (Pitx3) gene (base pairs 71-356; GenBank accession number AF005772), Otx2, and Fgf8 (Broccoli et al., 1999). Two micrograms of linearized plasmid were used as a template for the synthesis of radiolabeled transcripts by in vitro transcription with ${ }^{35}$ S-UTP. After $20 \mathrm{~min}$ of DNase I (Roche, Penzberg, Germany) treatment, the probes were purified by the RNeasy Clean up protocol (Qiagen, Hilden, Germany) and measured in a scintillation counter. For hybridization, sections were dewaxed, pretreated, and prehybridized as described previously (Dagerlind et al., 1992). Subsequently, they were hybridized overnight with a probe concentration of $7 \times 10^{7} \mathrm{cpm} / \mathrm{ml}$ at $57^{\circ} \mathrm{C}$ and washed at $65^{\circ} \mathrm{C}$ in $0.1 \times$ SSC and $0.1 \mathrm{~mm}$ DTT. The hybridized slides were dipped in autoradiographic emulsion (type NTB2; Eastman Kodak, Rochester, NY), developed after 3-6 weeks, and counterstained with cresyl violet. Deter- mination of the volume of expression domains was performed using the method of unbiased stereology, specifically the Cavalieri estimator of volume (Gundersen and Jensen, 1987) using an interactive computer system (KS400; Zeiss, Hallbergmoos, Germany). Whole-mount in situ hybridization was performed according to Broccoli et al. (1999).

Retrograde tracing. Six En1 ${ }^{+/ O t x 2}$ mutants and six matched wild-type littermates were used for this study. After deep anesthesia using a mixture of ketamine/rompun, animals were placed into a stereotaxic frame. Stereotaxic coordinates for injections were taken from the atlas of Franklin and and Paxinos (1997). After drilling a hole into the skull directly over the injection site, $2-3 \times 0.5 \mu$ l of a $2 \%$ fluorogold (FG) solution in water was pressure injected into the striatum using a Hamilton syringe. After injection, the injection cannula was left in place for at least 5 min to prevent spilling of the tracer along the injection tract. Surgery was finished by closing the skull with bone wax and sewing the scalp. At all times, animals were handled according to the Society for Neuroscience Policy on the Use of Animals in Neuroscience Research as well as the European Communities Council Directive.

After a survival time of $5 \mathrm{~d}$, the animals were again deeply anesthetized and perfused transcardially. Perfusion was performed for 2 min with 0.1 м PBS at room temperature, immediately followed by ice-cold $4 \%$ paraformaldehyde in $0.1 \mathrm{~m}$ PBS. After perfusion, the brains were removed from the skull, postfixed for $2 \mathrm{hr}$ in $4 \%$ paraformaldehyde, and cryoprotected in $20 \%$ sucrose in $0.1 \mathrm{M}$ PBS overnight. The brain was cut alternating into $1 \times 50$ - $\mu \mathrm{m}$-thick and $2 \times 30$ - $\mu \mathrm{m}$-thick horizontal frozen sections using a cryostat (Microm HM560). The 50- $\mu$ m-thick sections were processed for visualization of TH and dopamine transporter (DAT) by immunohistochemistry. The $30 \mu \mathrm{m}$ thick sections were processed for tracer visualization and simultaneous detection of tracer and $\mathrm{TH}$ as well as DAT.

Immunohistochemistry. Free-floating 50- $\mu \mathrm{m}$-thick sections and dissected neural tubes [embryonic day 10.5 (E10.5)] were rinsed in $0.1 \mathrm{M}$ PBS, preincubated for $1-2 \mathrm{hr}$ in $10 \%$ fetal calf serum in $0.1 \mathrm{M}$ PBS. Thereafter, the sections and neural tubes were incubated in primary antibody overnight at $4^{\circ} \mathrm{C}$ and constantly agitated (rabbit-anti-TH, 1:10,000; Pel-Freeze Biologicals, Rogers, AZ; rat-anti-DAT, 1:2000; Chemicon, Temecula, CA; mouse anti-Islet-1, 1:1000; Developmental Studies Hybridoma Bank, Iowa City, IA). Sections and neural tubes were then washed five times for $10 \mathrm{~min}$ in $0.1 \mathrm{M}$ PBS; incubated for $45 \mathrm{~min}$ in biotinylated anti-rabbit IgG (Vectastain, 1:300; Vector Laboratories, Burlingame, CA), biotinylated anti-rat IgG (Vectastain, 1:300), and biotinylated anti-mouse IgG (Vectastain, 1:300), respectively; washed five times for $10 \mathrm{~min}$ in $0.1 \mathrm{M} \mathrm{PBS}$, and incubated for $45 \mathrm{~min}$ in $\mathrm{ABC}$ solution (Vectastain, 1:300). After another $10 \mathrm{~min}$ wash in $0.1 \mathrm{M}$ PBS, sections were transferred to $0.1 \mathrm{M}$ Tris- $\mathrm{HCl}, \mathrm{pH} 7.2$, and immunohistochemistry was completed within 10-20 min using diaminobenzidine as a chromogen for visualization of the peroxidase reaction. Sections and flattened neural tubes were mounted on slides and embedded in ProTaq MountFluor (Quartett Immunodiagnostics, Berlin, Germany). Thirtymicrometer-thick sections were preincubated for $1-2 \mathrm{hr}$ in $10 \%$ FCS in $0.1 \mathrm{M}$ PBS. Thereafter, the sections were incubated in the dark in primary antibody overnight at $4^{\circ} \mathrm{C}$ (rabbit-anti-TH, 1:1000; Pel-Freeze Biologicals; rat-anti-DAT, 1:500; Chemicon). Sections were then washed five times for $10 \mathrm{~min}$ in $0.1 \mathrm{M}$ PBS, incubated for $1 \mathrm{hr}$ in Texas Red anti-rabbit IgG (Vectastain, 1:800) and Texas Red anti-rat IgG (Vectastain, 1:800), respectively, washed five times for $10 \mathrm{~min}$ in $0.1 \mathrm{M} \mathrm{PBS}$, and mounted in ProTaq.

NADPH-diaphorase histochemistry. Free-floating 50- $\mu$ m-thick sections were postfixed for $20 \mathrm{~min}$ in $4 \%$ PFA and washed three times for 5 $\min$ in $0.1 \mathrm{M} \mathrm{PBS}$. For staining, they were incubated at $37^{\circ} \mathrm{C}$ in staining solution (1.2 м $\beta$-NADPH, $0.1 \mathrm{mg} / \mathrm{ml}$ nitroblue tetrazolium, $0.3 \%$ Triton $\mathrm{X}-100$, and $1 \mathrm{~mm} \mathrm{MgCl}$ dissolved in $0.1 \mathrm{M} \mathrm{PBS}$ ). Finally, sections were washed three times for $10 \mathrm{~min}$ in $0.1 \mathrm{~m}$ PBS, mounted, dehydrated, and mounted in ProTaq Mount Fluor.

Transmitter measurement. Brains of mice were dissected and shock frozen on dry ice and cut on a cryostat in $200-\mu \mathrm{m}$-thick sections. Striatal tissue was recovered by punching the region of interest. DA and 5-HT content was measured by HPLC according to Sillaber et al. (1998) and referred to tissue weight to obtain transmitter concentrations. 




Figure 1. Shifting the $\mathrm{MHO}$ caudally in $E n 1^{+/ 0 t x 2}$ mutants enlarges the mid-DA neuronal population and decreases the rost5-HT cell group. mRNA in situ hybridization on consecutive sagittal sections of E12.5 wild-type (WT; $A-D)$ and En $1^{+/ 0 t x 2}$ transgenic embryos $(E-H)$ is shown. $A, E$, Bright-field images. $B-D, F-H$, Dark-field images of adjacent sections. $B, F$, The caudal limit of $0 t x 2$ expression marks the position of MHO (arrows). C, Wild-type mid-DA cells, marked by Dat expression, are located rostral to the MH0.D, Rost-5-HT cells, identified by Sert, are located caudal to the MHO.F-H, Shifting the MHO caudally in En $1^{+/ 0 t \times 2}$ littermates $(F)$ leads to an enlargement of the mid-DA cell population $(G)$, whereas the rost-5-HT neuronal population $(H)$ is complementary decreased. Each arrowhead indicates the original position of the MHO. MF, Mesencephalic flexure; III, third ventricle; Aq, Aqueduct; RP, Rathke's pouch.

Stereological analysis. Three animals of each group and each stage were analyzed. All volume measurements and cell counts were performed with the help of an interactive computer system (KS400; Zeiss). The volume of the SN [including the volume of the ectopic neurons in the paralemniscal area $(\mathrm{PL})$ ] was calculated using the method of Cavalieri. To determine the numerical density of TH-positive neurons, immunopositive neurons in this region were recorded using systematic random sampling and optical dissector methods. The total number of TH-positive neurons was calculated by referring their numerical density to the volume of the substantia nigra.

Activity measurements. All behavioral tests were performed between 9:00 A.M. and 1:00 P.M. To study motor activity, naive En1 $1^{+/ O t x 2}$ and wild-type littermates were placed in an open field $(30 \times 30 \mathrm{~cm}$; illumination, $30 \mathrm{lux}$ ), and locomotor activity was monitored by video-tracking for a period of $60 \mathrm{~min}$.

\section{Results \\ MHO positioning and mid-DA and rost-5-HT cell populations}

First, we analyzed $E n 1^{+/ O t x 2}$ mutants, in which one allele of the En1 coding sequence was replaced by an Otx2 minigene, leading to a caudal shift of the Otx2 expression domain into rhombomere 1 (rh1) (Broccoli et al., 1999). This shift, which is more pronounced dorsally, results in an enlargement of the inferior colliculi and a loss of the anterior vermis in the dorsal part of the midbrain-hindbrain region. However, no gross morphological alterations are detected ventrally (Broccoli et al., 1999).

To investigate whether the ventrally located mid-DA and rost5-HT transmitter populations are affected by the subtle ventrocaudal shift of the MHO, we studied the expression domains of specific markers on consecutive sections of mutants and wildtype littermates at E12.5 by in situ hybridization. At this stage, DA and 5-HT markers are already robustly expressed. DA neurons were identified by probes for Th, Dat, and the mid-DA neuronspecific marker Ptx3 (Smidt et al., 1997). 5-HT cells were marked by serotonin transporter (Sert) and Pet1 (Hendricks et al., 1999; Pfaar et al., 2002) expression.

In the wild-type littermates, the caudal edge of Dat (Fig. 1C) and $T h$ (data not shown) expression extended closely up to the caudal expression border of Otx2 (Fig. $1 B, C)$, with only a few cell layers spared. Also, in $E n 1^{+/ O t x 2}$ mutants, in which the MHO is shifted caudally, the posterior limit of the Th and Dat expression was close to the caudal Otx2 expression domain. This results in a posterior shift of the caudal edge of the Th and Dat expression area. Because the rostral edge of these expression domains remains unaltered, the dopaminergic cell population is enlarged (Fig. 1F,G). Rost-5-HT neurons are located immediately caudal to the Otx2 expression domain (Fig. $1 B, D$ ). Shifting the Otx2 expression caudally leads to a reduction in the rost-5-HT cell population (Fig. $1 \mathrm{H}$ ).

Quantification of the Dat and Sert expression domains in the midbrain-hindbrain region of wild-type and $E n 1^{+/ O t x 2}$ littermates revealed that the volume of the wild-type Dat expression domain was $12 \times 10^{6} \mu^{3}$ [average, $\pm 0.14 \times 10^{6}$ (SEM)], whereas it was $35.76 \times 10^{6} \mu \mathrm{m}^{3}$ [average, $\left.\pm 0.69 \times 10^{6}(\mathrm{SEM})\right]$ in the $E n 1^{+/ O t \times 2}$ mutants. This difference was highly significant ( $t$ test; $p=0.003$ ). On average, the volume of the Dat expression domain increased 2.98-fold [23.76 $\times 10^{6}$ $\mu \mathrm{m}^{3}$; average, $\left.\pm 0.55 \times 10^{6}(\mathrm{SEM})\right]$. The volume of the Sert expression domain in wild-type littermates was $50.76 \times 10^{6} \mu \mathrm{m}^{3}$ [average, $\pm 1.11 \times 10^{6}(\mathrm{SEM})$ ], whereas it was $29.37 \times 10^{6} \mu \mathrm{m}^{3}$ [average, $\left.\pm 0.76 \times 10^{6}(\mathrm{SEM})\right]$ in $E n 1^{+/ O t \times 2}$ mutants. Again, this difference was highly significant ( $t$ test; $p=0.003$ ). On average, the volume of the Sert expression domain decreased 0.58 -fold $\left[21.39 \times 10^{6} \mu^{3}{ }^{3}\right.$; average, $\left.\pm 0.55 \times 10^{6}(\mathrm{SEM})\right]$. These results indicate that the caudal shift of the MHO leads to an increase in the Dat expression domain that is equivalent to the decrease in the Sert expression domain.

To determine whether a rostral shift of the MHO would lead to a complementary change of the location and size of mid-DA and rost-5-HT cell populations, we investigated Otx1 ${ }^{-/}$; Otx $2^{+/-}$mutants. In these mutants, which have no functional Otx1 gene and only one intact Otx2 allele, the OTX protein levels are reduced under a critical threshold. As a consequence, the $\mathrm{MHO}$ is shifted rostrally close to the zona limitans intrathalamica within the diencephalon (Acampora et al., 1997; Suda et al., 1997). Otx $1^{-/-} ;$Otx $x 2^{+/-}$mutants were compared with Otx $2^{+/-}$ littermates, which are phenotypically wild type. The MHO was defined by the caudal border of Otx 2 and by F $g f$ expression (Fig. $2 B, C, E, F)$. We observed that cells expressing $T h$ were shifted rostrally into the diencephalic tissue immediately rostral to the ectopically positioned $\mathrm{MHO}$ (Fig. $2 G, J$ ). In addition, Ptx3, which is marking specific mesencephalic DA neurons at the exclusion of other DA neurons (Smidt et al., 1997), was also relocated rostrally (Fig. 2H,K). Complementary expression of the serotonergic marker Sert expanded up to the presumptive diencephalon posterior to the caudal Otx2 expression border. Because the caudal position of the 5-HT neurons was not altered, a considerable enlargement of this expression domain was observed (Fig. 2I,L).

To examine whether the early shift of the MHO in both mutants has a persistent effect on the location and extension of the two transmitter populations, we analyzed the expression of phenotypic markers $3 \mathrm{~d}$ later in development. In the $E n 1^{+/ O t \times 2} \mathrm{mu}-$ 
tants in which the MHO was shifted caudally, the mid-DA population is still expanded at E15.5 (Fig. 3A,C) and the rost-5-HT cell population remained significantly reduced (Fig. $3 B, D$ ). The decrease in the 5 -HT cell population primarily concerned the dorsal raphe (DR) nuclei (Fig. 3D), whereas the median raphe (MR) (Fig. 3D) was less affected. In Ot $x 1^{-/-} ;$Ot $x 2^{+-1}$ mutants, in which the $\mathrm{MHO}$ is shifted rostrally, a population of Dat-expressing cells can still be found at an ectopic position in the ventral diencephalon at E15.5, and the rost-5-HT cell population is expanded up to the caudal limit of the diencephalon (Fig. $3 E-H$ ). This indicates that the changes in the two transmitter populations induced by an aberrantly positioned MHO are maintained during embryogenesis.

To investigate whether the alterations in the location and number of mid-DA and rost-5-HT neurons observed during embryogenesis are still present in adulthood, we analyzed their location and extension in adult $E n 1^{+/ O t x 2}$ mice, which are, in contrast to Ot $x 1^{-/-} ;$Ot $x 2^{+/-}$mutants, viable and fertile. Consistent with the results obtained at embryonic stages, Th and Dat expression were expanded posteriorly (Fig. 4A-F), whereas the Sert and Pet1 expression domains were decreased in size. This decrease primarily affects the dorsal raphe nuclei (Fig. 4G-L).

To analyze the increased dopaminergic neuronal population in more detail, immunohistochemistry for $\mathrm{TH}$ and DAT was performed. A caudal extension of the dopaminergic cell group of the RrF could be observed, as well as the appearance of an ectopically located dopaminergic cell population in the PL as a caudal extension of the SN (Fig. 5A-D). Both ectopic cell populations stained for TH and DAT, indicating their dopaminergic identity (Fig. $5 B, D)$. For quantification, we specifically determined the volume of the $\mathrm{TH}$-positive neuronal population and their numerical density in the SN and its caudal extension. This was done because the $\mathrm{SN}$ is involved in the regulation of motor activity, which is increased in the mutants (see below). The volume of the SN marked by TH-positive cells in the wild-type mice was $724.70 \times 10^{6} \mu \mathrm{m}^{3}$ [average, $\pm 9.58 \times 10^{6}(\mathrm{SEM})$ ], whereas it was of $977.49 \times 10^{6}$ $\mu \mathrm{m}^{3}$ [average, $\left.\pm 45.61 \times 10^{6}(\mathrm{SEM})\right]$ in the mutant mice. The difference in the volumes was significant ( $t$ test; $p=0.0114$ ). The total number of TH-positive cells in the wild-type mice was 5390 [average, \pm 236 (SEM)], whereas it was 6424 [average, \pm 102 (SEM)] in the mutants. This increase in cell number was significant as well ( $t$ test; $p=0.0304$ ).

In addition, to determine whether the ectopically located DA neurons in the caudal extension of the SN innervate a proper DA target area, retrograde tracing studies were performed. A total of $1-1.5 \mu \mathrm{l}$ of FG was injected in vivo into the striatum to visualize efferent projections of the mesencephalic dopaminergic cell
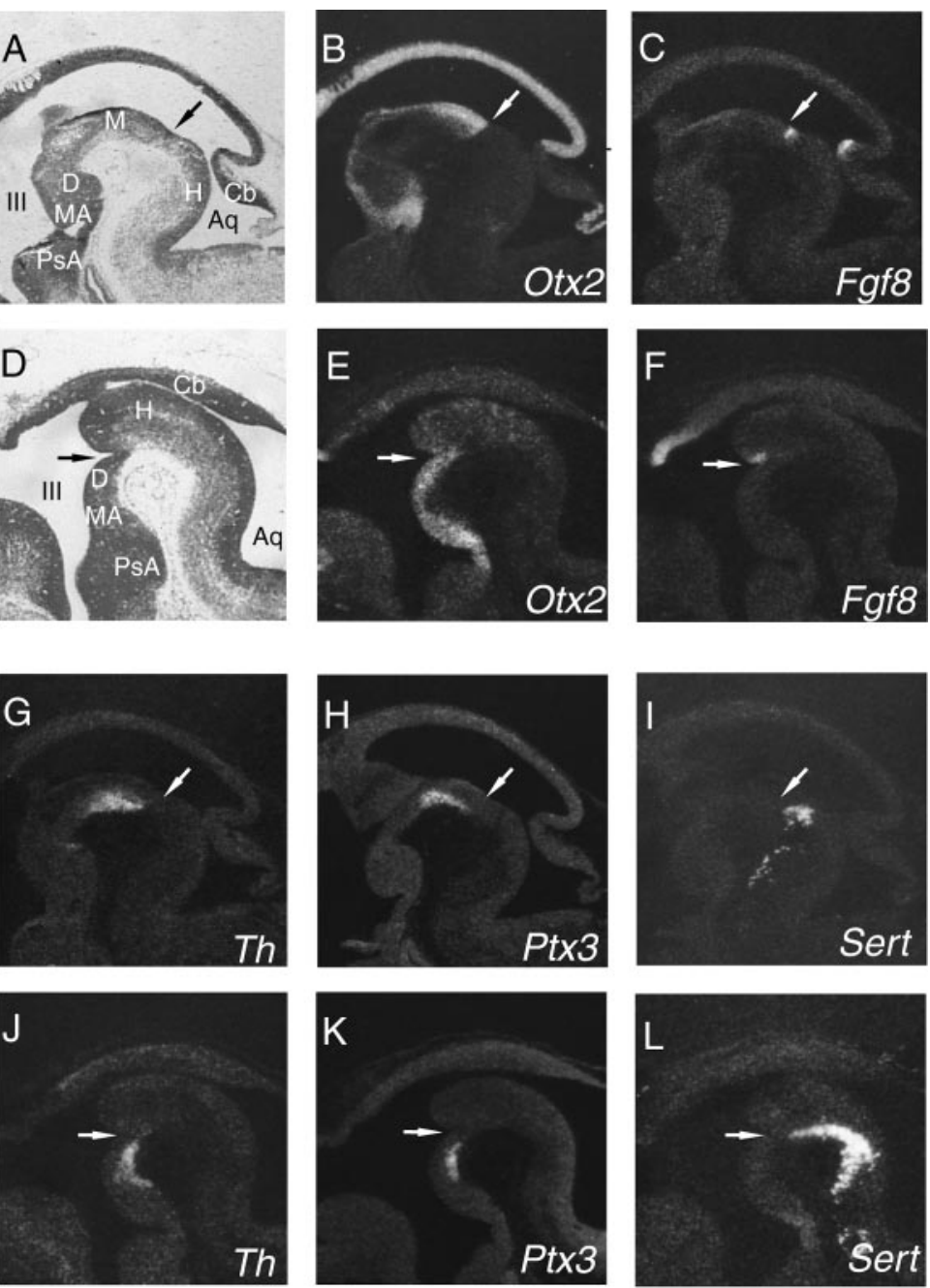

Figure 2. Shifting the $\mathrm{MHO}$ rostrally in $0 t \times 1^{-1-} ; 0 t \times 2^{+/-}$mutants relocates DA cells and enlarges the rost-5-HT neuronal population. mRNA in situ hybridization on consecutive sagittal sections of E12.5 Otx2 $2^{+/}$(phenotypically wild type) $(A-C, G-I)$ and $0 t \times 1^{-/-} ; 0 t \times 2^{+/-}(D-F, J-L)$ mutant embryos. In $0 t \times 1^{-1-} ; 0 t \times 2^{+/-}$mutants, the midbrain is missing and the enlarged caudal expression limit of $0 t \times 2(B, E)$ and by Fgf8 expression $(C, F)$ and is shifted rostrally in $0 t \times 1^{-/-} ; 0 t \times 2^{+/-}$mutants $(E, F) . G$, H, K, Mid-DA neurons marked by Th and Ptx3 are shifted rostrally to the ectopic MHO in mutant embryos. I, L, The rost-5-HT the position of the MHO. III, Third ventricle; Aq, Aqueduct; $\mathrm{Cb}$, cerebellum; $\mathrm{D}$, ventral diencephalon; $\mathrm{H}$, ventral hindbrain; $\mathrm{M}$, ventral midbrain; MA, mammillary area; PsA, Postoptic area.

groups. The FG injection involved major portions of the striatum and resulted in the retrograde labeling of many but not all DA neurons in the ipsilateral substantia nigra, the VTA, and the retrorubral field. In wild-type mice, no retrogradely labeled cells could be observed in the PL (data not shown). In contrast, in mutant mice, a substantial proportion of the ectopically located dopaminergic cells in this area were positive for FG, indicating that they do project to the striatum (Fig. 5G-I).

To investigate whether the changes in the size of mid-DA and rost-5-HT neuronal populations would lead to a change in the concentrations of neurotransmitters in a target area, we measured DA and 5-HT in the striatum of adult wild-type and $E n 1^{+/ O t x 2}$ mutants by HPLC. The DA concentration was significantly increased (Wilcoxon matched-pairs test; $n=6 ; p=0.028$ ) by $15.3 \%$ ( \pm SEM $7.8 \%$ ), whereas the 5 -HT concentration was decreased to $24.5 \%$ ( \pm SEM $4.6 \%$ ). This difference was significant as well (Wilcoxon matched-pairs test; $n=6 ; p=0.028$ ).

In summary, these results provide evidence that the position 


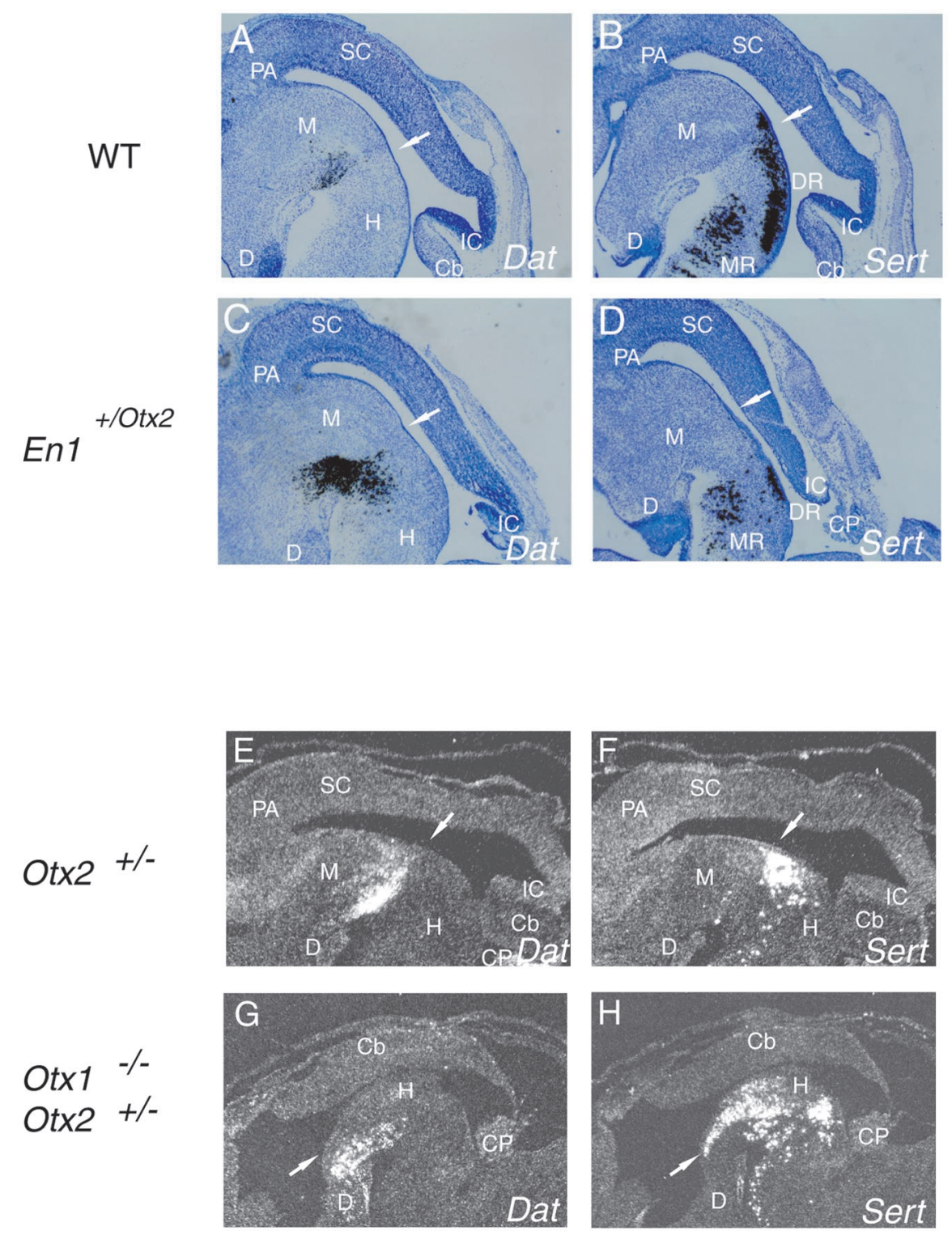

Figure 3. Changes in DA and 5-HT cell populations persist into later embryonic stages. mRNA in situ hybridization on consecutive sagittal sections of wild-type (WT) mice $(A, B)$ and their $E n 1^{+/ 0 t x z}$ littermates $(C, D)$ at E15.5 is shown. $A$, C, Shifting the MHO caudally leads to an enlargement of the DA cell population marked by Dat expression. $B, D$, The rost- 5 -HT neuronal population characterized by Sert mRNA is reduced in the mutants. The DR is more affected than the MR. E-H, mRNA in situ hybridization of consecutive sagittal sections of E15.5 $0 \mathrm{tx} 2^{+/-}$embryos (phenotypically wild type) and $0 \mathrm{t} \times 1^{-1-} ; 0 \mathrm{tx} 2^{+/-}$mutants. In Otx $1^{-/-} ; 0 t \times 2^{+/-}$mutants in which the MHO is shifted rostrally, a population of Dat-expressing cells can still be found at an ectopic position in the ventral diencephalon $(E, G)$, and the rost-5-HT cell population is expanded up to the caudal limit of the diencephalon and enlarged $(F, H)$. Each arrow indicates residual of isthmic fossa. Cb, Cerebellum; $C P$, choroid plexus; $D$, ventral diencephalon; $\mathrm{M}$, ventral midbrain; $\mathrm{H}$, ventral hindbrain; IC, inferior colliculus; $\mathrm{SC}$, superior colliculus; $\mathrm{PA}$, pretectal area.

of the MHO, which is determined in embryogenesis by the Otx2/ Gbx2 interaction (Broccoli et al., 1999; Millet et al., 1999), defines the extension of territories giving rise to mid-DA and rost-5-HT cell populations and controls their location and size in vivo. In addition, the ectopically located mid-DA neurons in the diencephalon of $\mathrm{Otx} 1^{-/-} ; \mathrm{Otx} 2^{+/-}$mutants suggest that signals from the $\mathrm{MHO}$ are sufficient to induce dopaminergic neurons in vivo.

\section{MHO positioning and ventral} midbrain-hindbrain structures

Here we report an expansion of the mid-DA cell population and a decrease in the rost-5-HT cell group in En1 ${ }^{+/ O t x 2}$ mutants. To study whether the positioning of the MHO similarly affects other ventral midbrain-hindbrain neuronal populations, we analyzed nuclei adjacent to these populations, such as the cranial nerves III, IV, and V and the nucleus pedunculopontinus.

To determine whether the ectopic dopaminergic neurons in the adult are indeed located in the rostral hindbrain rather than in a transformed midbrain, we studied the identity of the surrounding tissue in the PL of En1 $1^{+/ O t x 2}$ mutants. For this purpose, we examined the presence of the pedunculopontine nucleus, which is located in the PL. NADPH-diaphorase histochemistry revealed the preserved identity of this nucleus (Fig. $5 D, F$ ). Thus the ectopic DA neurons are located in the rostral hindbrain.

The nucleus occulomotorius (cranial nerve III) develops immediately rostral to the MHO adjacent to the mid-DA neurons, whereas the nuclei trochlearis and trigeminalis (cranial nerves IV and V) develop caudal to it at the same anteroposterior level as the rost-5-HT neurons. The analysis of Islet-1 staining in En1 $1^{+/ O t x 2}$ mutants at E10.5 shows that the nucleus occulomotorius is not expanded to the caudal Otx2 border and enlarged, as is the case for the dopaminergic neuronal population (Fig. 5J; compare Fig. $1 C, G$ ). In addition, the nuclei trochlearis and trigeminalis are not altered, corresponding to the change in the rost-5-HT cell group (Fig. $5 J$; compare Fig. $1 D, H)$. In $O t x 1^{-/-}$; Otx $2^{+/-}$mutants at E12.5, the nuclei trochlearis and trigeminalis were relocated at the newly positioned MHO but did not change in size (data not shown).

Together, our results show that other cell populations in the ventral midbrain-hindbrain region are not affected similarly to the mid-DA and 5-HT neurons, suggesting that the position of the MHO has different effects on distinct cell populations.

\section{MHO positioning and adult behavior}

Mid-DA and rost-5-HT neurons modulate different aspects of adult behavior (Cooper et al., 2001), such as motor activity (Zhou and Palmiter, 1995; Jacobs and Fornal, 1997). Because the position of the MHO defines the size ratio between these populations, we hypothesized that an aberrant position of the MHO in En1 $1^{+/ O t x 2}$ mutants would lead to a change in their motor activity. This would indicate that the position of the MHO during embryogenesis is of functional relevance for adult behavior.

Locomotor activity of $E n 1^{+/ O t x 2}$ mutants and wild-type mice was studied in an open field by video-tracking. A highly significant enhancement of locomotion in $E n 1^{+/ O t x 2}$ mice compared with their wild-type littermates $\left(F_{(1,47)}=22.95 ; p<0.00001\right)$ independent of gender (genotype $\times$ gender interaction: $F_{(1,47)}=$ $0.32 ; p=0.57$ ) was revealed by multivariate ANOVA (Fig. 6).

Atactic behavior of $E n 1^{+/ O t \times 2}$ mutants has been described pre- 
viously (Broccoli et al., 1999) and was attributed to the cerebellar defect. Therefore, to investigate whether there is a correlation between the ataxia and the locomotor activity, we tested the animals of the open-field study by means of the rotarod. However, no correlation between atactic behavior and locomotor activity was observed (data not shown).

\section{Discussion}

MHO positioning and development of mid-DA and rost-5-HT

neuronal populations

The position of the MHO is determined during embryogenesis by the interaction of Otx2 and Gbx2 (Broccoli et al., 1999; Millet et al., 1999). Our results suggests that Otx2 is an important upstream signal for mid-DA specification and $G b \times 2$ is important for rost-5-HT development. Although we cannot exclude the possibility that these two genes are directly involved in the development of these cell populations, it is more likely that signals generated at the $\mathrm{MHO}$, such as $F g f 8$, are mediating these effects (Ye et al., 1998). In addition, Shh is necessary for the induction of mid-DA and rost-5-HT neurons in an explant culture system (Ye et al., 1998). However, both transmitter populations are equally dependent on Fgf8 and Shh, suggesting that there must be additional signals specifying DA versus 5-HT identity. In En $1^{+/ O t x 2}$ mutants, the shift of the MHO leading to a change in the location and extension of mid-DA and rost-5-HT neuronal populations takes place after the onset of somitogenesis (E8). This indicates that these additional signals are generated within the neural epithelium and are most likely emitted by the MHO after this time point. The results presented here also suggest that genes expressed at the MHO, such as En1, En2, Wnt1, Pax2, Pax5, Pax8, Fgf17, Fgf18, Lmx 1b, and Ptx3 (McMahon et al., 1992; Millen et al., 1994; Wurst et al., 1994; Schwarz et al., 1997; Smidt et al., 1997, 2000), might be involved in the specification of DA versus 5-HT neurons. It has been shown that En1 and En2 are necessary for the maintenance but not for the specification of mid-DA neurons in a gene dose-dependent manner (Simon et al., 2001). Together, the results suggest that Otx2 determines the position of a genetic cascade involving $F g f 8, E n 1, E n 2$, and other unidentified genes expressed at the MHO together with Shh to control the localization, number, and survival of mid-DA neurons. In contrast, $G b \times 2$ positions together with $O t \times 2$, a molecular network including $F g f 8$, and yet undefined signals from the MHO that regulate together with Shh the development of rost5-HT neurons.

An unexpected finding of this study was that the mid-DA and
WT
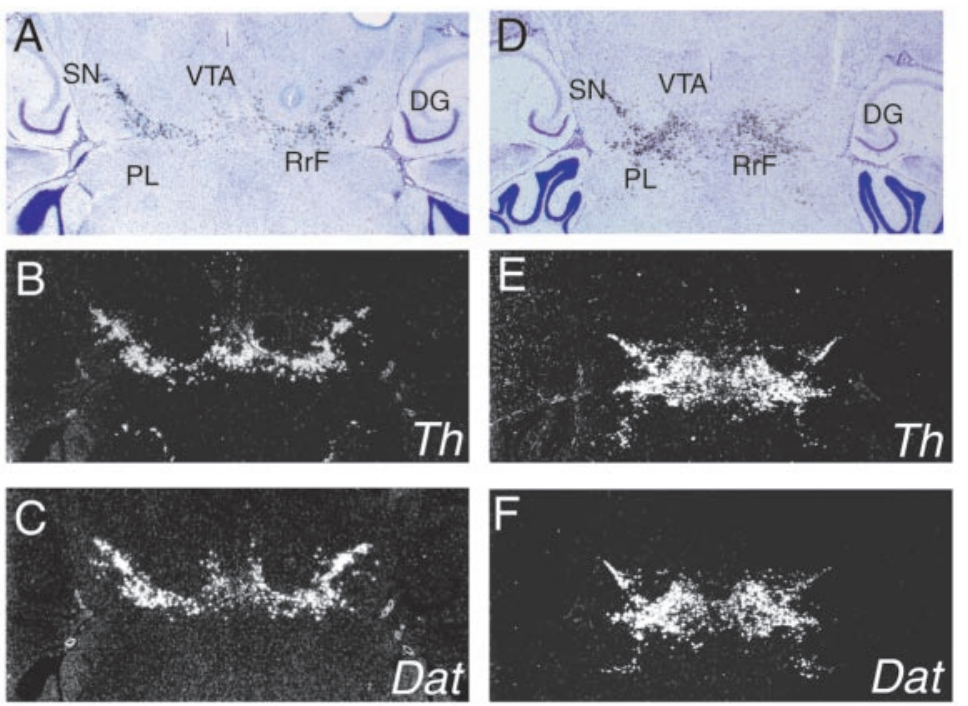
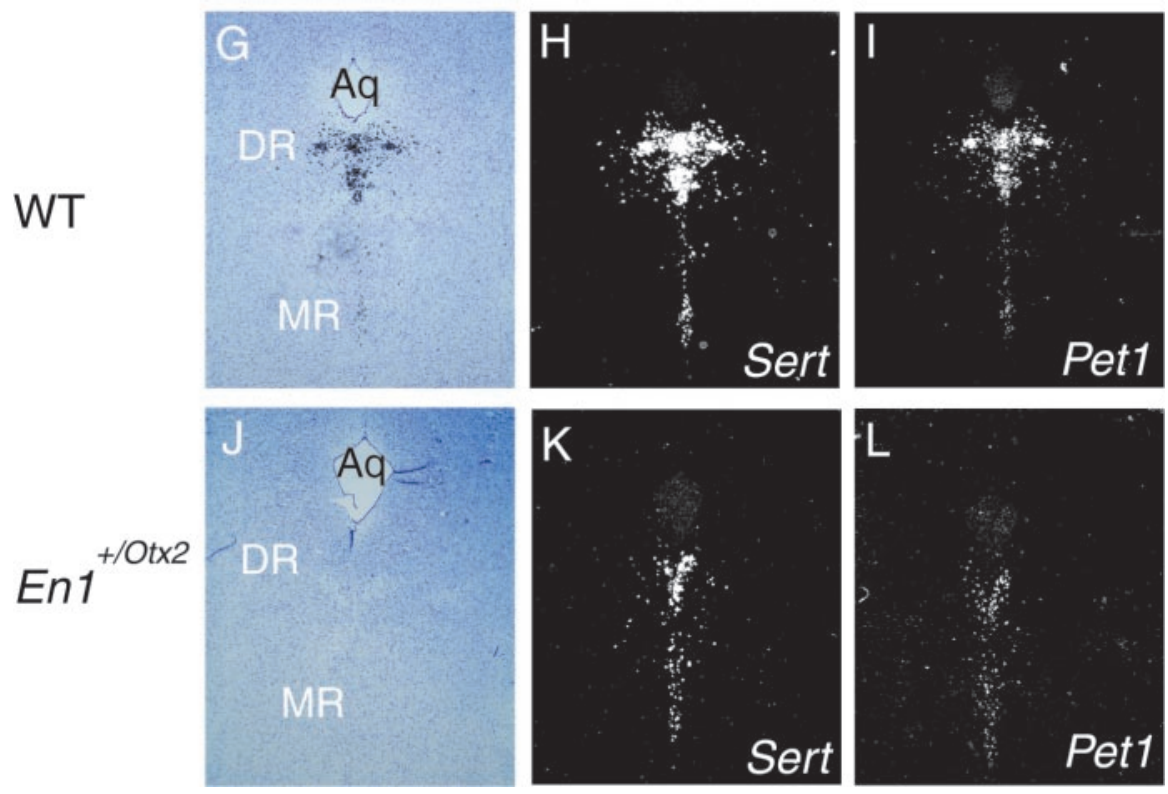

Figure 4. Alterations in $E n 1^{+10 t \times 2}$ mutants are maintained in adulthood. mRNA in situ hybridization on consecutive sections of adult wild-type (WT) mice $(A-C, G-I)$ and $E n 1^{+10 t x 2}$ mutants $(D-F, J-L)$. $A-F$, horizontal sections. $A, D$, Bright-field image of $D a t$. $B, C, E, F$, Dark-field image of Th and Dat. Both expression domains mark the substantia nigra, ventral tegmental area, and retrorubral field. DA markers are found more caudally in adult $E n 1^{+/ 0 t x 2}$ mice compared with wild-type mice. $G-L$, Coronal sections (anteroposterior level: Bregma, $-4.36 \mathrm{~mm}$ ). G, J, Bright-field image of same slide as the dark-field image of Sert shown in $\mathrm{H}$ and $K)$. En $1^{+10 t x 2}$ mutants $(J-L)$ show reduced Sert and Pet 1 expression compared with wild types $(G-l)$, predominantly in the DR. Aq, Aqueduct; DG, dentate gyrus.

rost-5-HT cell populations were not only relocated in mutants in which the MHO was shifted, but also changed in size, depending on the position of the $\mathrm{MHO}$ (Fig. 7). This suggests that the $\mathrm{MHO}$ is not only an anatomical hallmark but could actively influence the function of these two neuronal populations by defining their size through its location.

Although the MHO is shifted caudally in the En1 $1^{+/ O t x 2}$ mice and the size ratio between mid-DA and rost-5-HT cell populations is altered, the rostral limit of the mid-DA and the caudal border of the rost-5-HT neurons remains unaltered. This suggests that there must be additional signals cooperating with those 

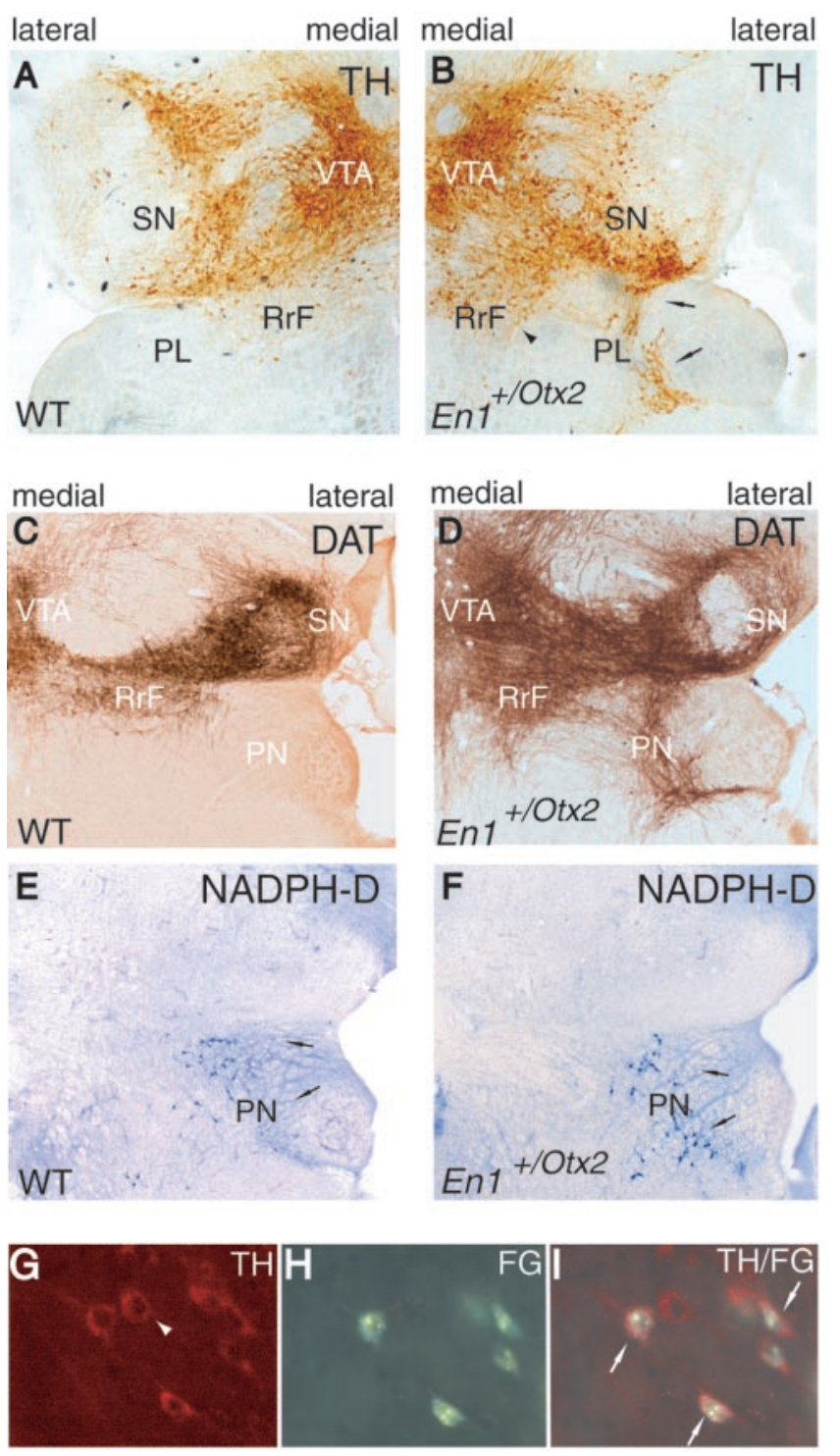

J

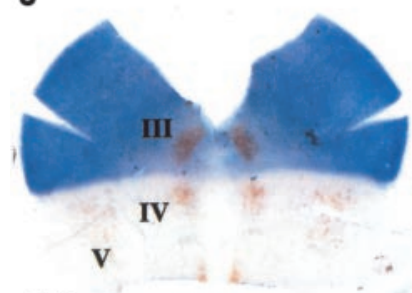

WT

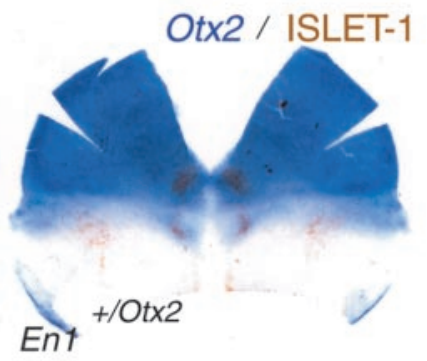

Figure 5. Ventral midbrain-hindbrain structures and projections of ectopic dopaminergic neurons in $E n 1^{+/ 0 t \times 2}$ mutants. $A, B$, TH immunohistochemistry on horizontal sections. Shown here is the left half of the ventral midbrain- hindbrain region of adult wild-type (WT) mice $(A)$ and the right half of the ventral midbrain-hindbrain region of adult $E n 1^{+10 t \times 2}$ mice $(B)$. Ectopic TH-positive neurons are located caudally to the RrF (arrowhead) and SN (arrows). The latter are located in the PL. C, D, DAT immunohistochemistry marking dopaminergic neurons. E, F, $\mathrm{NADPH}$ - diaphorase histochemistry identifying the pedunculopontine nucleus (PN) on horizontal sections. Shown here are the right halves of the ventral midbrain- hindbrain region of adult mice. D, F, Adjacent sections of DAT and NADPH- diaphorase (NADPH-D) staining demonstrating the presence of the pedunculopontine nucleus around the ectopic DA neurons and indicating the preserved hindbrain identity of the tissue surrounding the ectopic cells. G-I, Cells in the PL of $E n 1^{+/ 0 t x 2}$ mutants. G, TH-positive neurons in the PL. H, After an injection of FG into the striatum, retrograde labeled cells are found in the PL. I, Overlay image showing that most $\mathrm{TH}$-positive cells are retrogradely labeled (arrows). However, some TH-positive neurons were
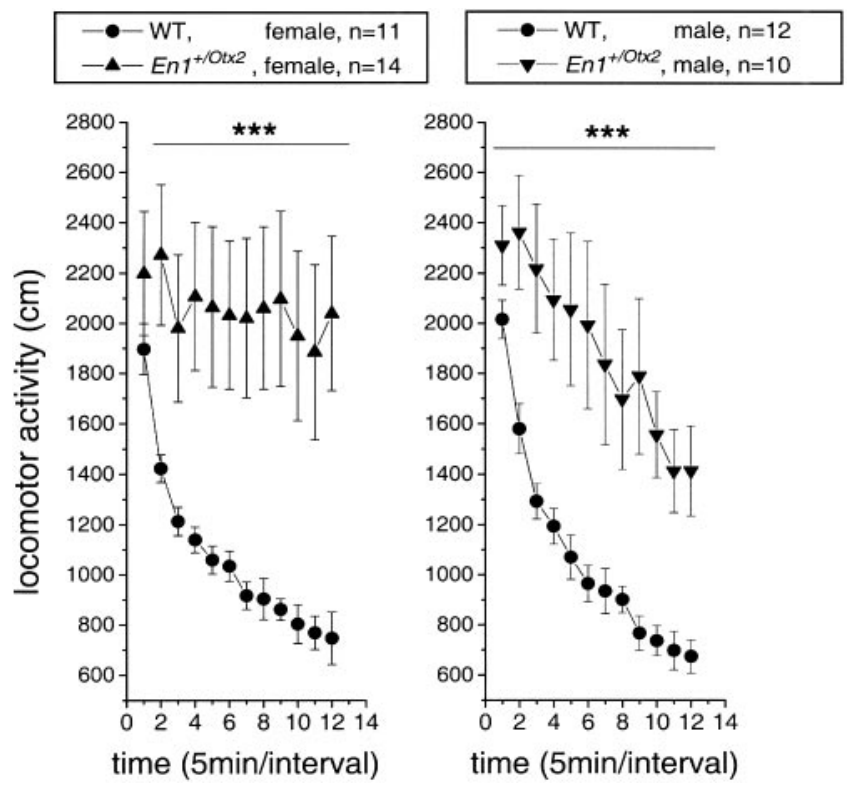

Figure 6. $E n 1^{+10 t x 2}$ mutants are hyperactive. Locomotor activity of $E n 1^{+10 t x 2}$ mice in an open field is shown. $E n 1^{+/ 0 t x 2}$ mice and their wild-type (WT) littermates were placed in an open field, and locomotor activity was monitored by video-tracking. En $1^{+10 t \times 2}$ mice showed enhanced locomotor activity (factor genotype, $\left.{ }^{* * *} p<0.00001\right)$ independent of gender $(p=0.57)$.

generated by the MHO to define the most anterior location of the mid-DA neurons as well as the most posterior location of the rost-5-HT neurons.

In Ot $x 1^{-/-} ; \mathrm{Ot} x 2^{+/-}$mutants, the $\mathrm{MHO}$ is shifted rostrally, in contrast to the $E n 1^{+/ O t x 2}$ mice, and to a greater extent beyond the original anterior limit of the mid-DA cell population. The mid-DA cell group is relocated rostral to the newly positioned MHO at an ectopic position in the diencephalon, without changing in size, but still expresses the midbrain DA-specific marker Ptx3. This suggests that the MHO is not dependent on other signals to induce mid-DA neurons. Consistent with the observations in the En1 $1^{+/ O t x 2}$ mutants, the caudal limit of the rost-5-HT cell population remains fixed. This indicates that here also, additional signals (in this case from the posterior hindbrain) determine the posterior extension of the rost-5-HT cell population.

An obvious difference between the mutants is that in $\mathrm{Ot} x 1^{-/-}$; $O t \times 2^{+/-}$mice, the newly positioned mid-DA cell population does not change in size, whereas in $E n 1^{+/ O t x 2}$ mice, the rost-5-HT cell group is decreased. This suggests that the anterior part of the neuroepithelium is permissive for the induction of mid-DA neuronal specification, whereas its posterior part is not entirely permissive for signals of the MHO inducing the development of rost-5-HT neurons.

Together, this suggests that the position of the MHO determines the size ratio between the mid-DA and rost-5-HT cell populations and is sufficient to induce at least the mid-DA neurons in the anterior neural epithelium. In addition, as yet unidentified signals emitted from the forebrain, possibly from the zona limi-

not (arrowhead). J, Dorsally opened and flattened anterior neural tube of E10.5 embryos. Double labeling with Otx2 whole-mount in situ hybridization and Islet-1 immunohistochemistry, marking the cranial nerves III, IV, and V. The nucleus occulomotorius (III) is not expanded to the caudal 0 tx2 border and enlarged, as is the case for the dopaminergic neuronal population (compare Fig. 1C, G). In addition the nuclei trochlearis (IV) and trigeminalis (V) are not altered, corresponding to the change in the rost-5-HT cell group (compare Fig. 1D, H). 



Figure 7. Influence of the position of the $\mathrm{MHO}$ on mid-DA and rost-5-HT cell populations. $A$, Mid-DA neurons develop rostral to the MHO in the Otx2 domain, and rost-5-HT cells develop caudal to it in the $G b \times 2$ expression region. $B, \ln E n 1^{+10 t x 2}$ mutants, $0 t \times 2$ and subsequently the MHO is shifted caudally (Broccoli et al., 1999) within the designated rost-5-HT region into rh1. This leads to an increase in the area of the mid-DA neuronal population and to a complementary decrease in the rost-5-HT cell group, indicating that the position of the MHO determines the size ratio between these two cell populations. $C, \ln 0 t \times 1^{-1-} ; 0 t \times 2^{+/-}$mutants, the reduced OTX protein levels induce a shift of the MHO (Acampora et al., 1997; Suda et al., 1997) rostral to the designated mid-DA region between $\mathrm{p} 2$ and $\mathrm{p} 3$ of the forebrain. Mid-DA neurons are induced rostral to the ectopically positioned $\mathrm{MHO}$, and the rost-5-HT cell population is enlarged. This suggests that the $\mathrm{MHO}$ is sufficient to induce mid-DA neurons along the anteroposterior axis and confirms the observations from the $E n 1^{+/ 0 t \times 2}$ mutants that the size ratio between the mid-DA and the rost- 5 -HT neurons is determined by the position of the MHO. WT, Wild type.

tans intrathalamica or the anterior neural ridge, cooperate with the MHO to define the rostral border of the area of mid-DA neurons. Accordingly, signals generated in the hindbrain might determine the caudal border of the rost-5-HT neuronal population.

\section{MHO positioning and ventral} midbrain-hindbrain structures

Mid-DA and rost-5-HT neurons are part of the basal plate of the developing neural tube. The changes reported here regarding the location and size of these cells in the studied mutants raise the question of whether a shift of the MHO specifically affects these transmitter populations or whether the shift transforms the region as a whole and these neurons are part of these alterations. Our analysis of other cell populations in the basal plate, such as the cranial nerves and the pedunculopontine nucleus, suggests that the $\mathrm{MHO}$ does indeed have a more direct effect on mid-DA and rost-5-HT neurons.

In Otx $1^{-/-} ; O t \times 2^{+/-}$mutants, the genetic network implicated in the maintenance of the $\mathrm{MHO}$ is shifted rostrally, leading to a complete transformation of the midbrain into hindbrain. Consistently in $E n 1^{+/ O t x 2}$ mutants, the caudal shift of Otx2 leads to a caudal shift of the genetic cascade and dorsally to a complete morphological transformation. Interestingly, in the ventral aspect of the midbrain-hindbrain region of the $E n 1^{+/ O t x 2}$ mutants, the changes in cell identity are obviously restricted to specific neuronal populations. This suggests that in this case, the underlying genetic network may not be temporally and/or spatially adequately repositioned. Alternatively, it suggests that different cell populations in the midbrain-hindbrain region depend on different genetic signals emitted by the $\mathrm{MHO}$ or are, however less likely, independent of it. Additional analysis will reveal which signals are implicated in the development of different neuronal populations.

\section{Influence of MHO positioning on adulthood}

The main focus of this study was to investigate the role of the position of the MHO for the embryonic development of mid-DA and rost-5-HT neurons. In contrast to Otx $1^{-/-} ; \mathrm{Ot} \times 2^{+/-} \mathrm{mu}-$ tants, $E n 1^{+/ O t x 2}$ mice are viable and fertile. Therefore, they also offer the possibility of analyzing the influence of the location of the $\mathrm{MHO}$ on adult mid-DA and rost-5-HT neurons as well as on behavior.

Here we report that in $E n 1^{+/ O t x 2}$ mutants, the increase in the size of the mid-DA and the reduction in the rost-5-HT cell populations persist into adulthood. This suggests that the location of the MHO could be a way of modulating the function of these transmitter systems in adulthood by regulating their location and size during development. If this hypothesis is true, the additional neurons should innervate a proper DA target area and the mutants should show an altered behavior. Indeed, we found that additional DA neurons project to the striatum, and that mutants with a caudally shifted $\mathrm{MHO}$ are hyperactive.

To investigate whether $E n 1^{+/ \text {otx } 2}$ mutants show a change in behavior, we decided to study the motor activity of these mice as it is influenced by the DA and 5-HT system (Zhou and Palmiter, 1995; Jacobs and Fornal, 1997). The observed hyperactivity is in accordance with previous studies. Pharmacological experiments have demonstrated that dopamine receptor stimulation is correlated with motor activity (Clark and White, 1987). These findings were supported by genetic experiments (Zhou and Palmiter, 1995; Giros et al., 1996; Gainetdinov et al., 1999). The inactivation of the dopamine transporter gene (Dat) leads to an increased synaptic dopamine concentration causing hyperactivity (Giros et al., 1996; Gainetdinov et al., 1999) and is regarded as a model for ADHD (Gainetdinov et al., 1999). The hyperlocomotion of these mutants can be antagonized by serotonergic activity, indicating that an imbalance between these two transmitter systems is responsible for this phenotype (Gainetdinov et al., 1999). En1 ${ }^{+/ O t x 2}$ mutants have other alterations in addition to changed mid-DA and rost-5-HT neuronal populations, such as an enlarged inferior colliculi and a loss of the vermis (Broccoli et al., 1999). In humans, hypoplasia of the posterior vermis has been implicated in ADHD (Castellanos and Tannock, 2002), which makes it conceivable that the missing vermis in the En1 $1^{+/ O t x 2}$ mice may account for the hyperactivity. However, the performed rota-rod test suggests that there is no correlation between atactic behavior, which would rather be attributed to a cerebellar defect, and the observed hyperactivity. Therefore, it seems unlikely that the cerebellar defect in the $E n 1^{+/ O t x 2}$ mice is associated with their hyperactivity. Although we cannot exclude the possibility that other changes in these mutants contribute to the altered locomotor activity, it seems most likely that this altered behavior is caused by increased DA and decreased 5-HT activity.

Together, we provide evidence that the position of the MHO, which is determined during embryogenesis, regulates the location and size of mid-DA and rost-5-HT neuronal populations. In addition, we suggest that it is of functional relevance for adult behavior. 


\section{References}

Acampora D, Avantaggiato V, Tuorto F, Simeone A (1997) Genetic control of brain morphogenesis through Otx gene dosage requirement. Development 124:3639-3650

Barr CL, Xu C, Kroft J, Feng Y, Wigg K, Zai G, Tannock R, Schachar R, Malone M, Roberts W, Nothen MM, Grunhage F, Vandenbergh DJ, Uhl G, Sunohara G, King N, Kennedy JL (2001) Haplotype study of three polymorphisms at the dopamine transporter locus confirm linkage to attention-deficit/hyperactivity disorder. Biol Psychiatry 49:333-339.

Björklund A, Lindvall O (1984) Dopamine containing systems in the CNS. In: Handbook of chemical neuroanatomy, Vol II, Classical transmitter in the CNS, Pt I (Björklund A, Hökfeld, T, eds), pp 55-122. Amsterdam: Elsevier.

Broccoli V, Boncinelli E, Wurst W (1999) The caudal limit of Otx2 expression positions the isthmic organizer. Nature 401:164-168.

Castellanos FX, Tannock R (2002) Neuroscience of attention-deficit/hyperactivity disorder: the search for endophenotypes. Nat Rev Neurosci 3:617-628.

Clark D, White FJ (1987) D1 dopamine receptor-the search for a function: a critical evaluation of the D1/D2 dopamine receptor classification and its functional implications. Synapse 1(4):347-388.

Cooper JR, Bloom FE, Roth RH (2001) The biochemical basis of neuropharmacology. New York: Oxford UP.

Dagerlind A, Friberg K, Bean AJ, Hokfelt T (1992) Sensitive mRNA detection using unfixed tissue: combined radioactive and non-radioactive in situ hybridization histochemistry. Histochemistry 98:39-49.

Favor J, Sandulache R, Neuhauser-Klaus A, Pretsch W, Chatterjee B, Senft E, Wurst W, Blanquet V, Grimes P, Sporle R, Schughart K (1996) The mouse Pax2(1Neu) mutation is identical to a human PAX2 mutation in a family with renal-coloboma syndrome and results in developmental defects of the brain, ear, eye, and kidney. Proc Natl Acad Sci USA 93:13870-13875.

Franklin KBJ, Paxinos G (1997) The mouse brain in sterotaxic coordinates (Franklin KBJ, Paxinos G, eds). San Diego: Academic.

Gainetdinov RR, Wetsel WC, Jones SR, Levin ED, Jaber M, Caron MG (1999) Role of serotonin in the paradoxical calming effect of psychostimulants on hyperactivity. Science 283:397-401.

Garda AL, Echevarria D, Martinez S (2001) Neuroepithelial co-expression of Gbx2 and Otx2 precedes Fgf8 expression in the isthmic organizer. Mech Dev 101:111-118.

Giros B, Jaber M, Jones SR, Wightman RM, Caron MG (1996) Hyperlocomotion and indifference to cocaine and amphetamine in mice lacking the dopamine transporter. Nature 379:606-612.

Goridis C, Rohrer H (2002) Specification of catecholaminergic and serotonergic neurons. Nat Rev Neurosci 3:531-541.

Gundersen HJ, Jensen EB (1987) The efficiency of systematic sampling in stereology and its prediction. J Microsc 147:229-263.

Hendricks T, Francis N, Fyodorov D, Deneris ES (1999) The ETS domain factor Pet-1 is an early and precise marker of central serotonin neurons and interacts with a conserved element in serotonergic genes. J Neurosci 19:10348-10356.

Hidalgo-Sanchez M, Simeone A, Alvarado-Mallart RM (1999) Fgf8 and Gbx2 induction concomitant with Otx2 repression is correlated with midbrainhindbrain fate of caudal prosencephalon. Development 126:3191-3203.

Jacobs BL, Fornal CA (1997) Serotonin and motor activity. Curr Opin Neurobiol 7:820-825.

Katahira T, Sato T, Sugiyama S, Okafuji T, Araki I, Funahashi J, Nakamura H (2000) Interaction between Otx2 and Gbx2 defines the organizing center for the optic tectum. Mech Dev 91:43-52.

Li JY, Joyner AL (2001) Otx2 and Gbx2 are required for refinement and not induction of mid-hindbrain gene expression. Development 128:4979-4991.

Marin F, Puelles L (1994) Patterning of the embryonic avian midbrain after experimental inversions: a polarizing activity from the isthmus. Dev Biol 163:19-37.

Martinez S, Alvarado-Mallart RM (1990) Expression of the homeobox chicken gene in chick/quail chimeras with inverted mes-metencephalic grafts. Dev Biol 139:432-436.

Martinez S, Wassef M, Alvarado-Mallart RM (1991) Induction of a mesencephalic phenotype in the 2-day-old chick prosencephalon is preceded by the early expression of the homeobox gene en. Neuron 6:971-981.

Martinez-Barbera JP, Signore M, Boyl PP, Puelles E, Acampora D, Gogoi R,
Schubert F, Lumsden A, Simeone A (2001) Regionalisation of anterior neuroectoderm and its competence in responding to forebrain and midbrain inducing activities depend on mutual antagonism between OTX2 and GBX2. Development 128:4789-4800.

McMahon AP, Joyner AL, Bradley A, McMahon JA (1992) The midbrainhindbrain phenotype of Wnt-1-/Wnt-1-mice results from stepwise deletion of engrailed-expressing cells by 9.5 days postcoitum. Cell 69:581-595.

Meyers EN, Lewandoski M, Martin GR (1998) An Fgf8 mutant allelic series generated by Cre- and Flp-mediated recombination. Nat Genet 18:136-141.

Millen KJ, Wurst W, Herrup K, Joyner AL (1994) Abnormal embryonic cerebellar development and patterning of postnatal foliation in two mouse Engrailed-2 mutants. Development 120:695-706.

Millet S, Campbell K, Epstein DJ, Losos K, Harris E, Joyner AL (1999) A role for Gbx2 in repression of Otx 2 and positioning the $\mathrm{mid} /$ hindbrain organizer. Nature 401:161-164.

Pfaar H, von Holst A, Vogt Weisenhorn DM, Brodski C, Guimera J, Wurst W (2002) mPet-1, a mouse ETS-domain transcription factor, is expressed in central serotonergic neurons. Dev Genes Evol 212:43-46.

Quist JF, Barr CL, Schachar R, Roberts W, Malone M, Tannock R, Basile VS, Beitchman J, Kennedy JL (2000) Evidence for the serotonin HTR2A receptor gene as a susceptibility factor in attention deficit hyperactivity disorder (ADHD). Mol Psychiatry 5:537-541.

Schwarz M, Alvarez Balado G, Urbanek P, Busslinger M, Gruss P (1997) Conserved biological function between Pax- 2 and Pax -5 in midbrain and cerebellum development: evidence from targeted mutations. Proc Natl Acad Sci USA 94:14518-14523.

Sillaber I, Montkowski A, Landgraf R, Barden N, Holsboer F, Spanagel R (1998) Enhanced morphine-induced behavioural effects and dopamine release in the nucleus accumbens in a transgenic mouse model of impaired glucocorticoid (type II) receptor function: influence of long-term treatment with the antidepressant moclobemide. Neuroscience 85:415-425.

Simeone A (2000) Positioning the isthmic organizer where Otx2 and Gbx2 meet. Trends Genet 16:237-240.

Simon HH, Saueressig H, Wurst W, Goulding MD, O’Leary DD (2001) Fate of midbrain dopaminergic neurons controlled by the engrailed genes. J Neurosci 21:3126-3134.

Smidt MP, van Schaick HS, Lanctot C, Tremblay JJ, Cox JJ, van der Kleij AA, Wolterink G, Drouin J, Burbach JP (1997) A homeodomain gene Ptx3 has highly restricted brain expression in mesencephalic dopaminergic neurons. Proc Natl Acad Sci USA 94:13305-13310.

Smidt MP, Asbreuk CH, Cox JJ, Chen H, Johnson RL, Burbach JP (2000) A second independent pathway for development of mesencephalic dopaminergic neurons requires Lmxlb. Nat Neurosci 3:337-341.

Suda Y, Matsuo I, Aizawa S (1997) Cooperation between Otx1 and Otx2 genes in developmental patterning of rostral brain. Mech Dev 69:125-141.

Tork I (1990) Anatomy of the serotonergic system. Ann NY Acad Sci 600:9-34.

Waldman ID, Rowe DC, Abramowitz A, Kozel ST, Mohr JH, Sherman SL, Cleveland HH, Sanders ML, Gard JM, Stever C (1998) Association and linkage of the dopamine transporter gene and attention-deficit hyperactivity disorder in children: heterogeneity owing to diagnostic subtype and severity. Am J Hum Genet 63:1767-1776.

Wassef M, Joyner AL (1997) Early mesencephalon/metencephalon patterning and development of the cerebellum. Perspect Dev Neurobiol 5:3-16.

Wurst W, Bally-Cuif L (2001) Neural plate patterning: upstream and downstream of the isthmic organizer. Nat Rev Neurosci 2:99-108.

Wurst W, Auerbach AB, Joyner AL (1994) Multiple developmental defects in Engrailed-1 mutant mice: an early mid-hindbrain deletion and patterning defects in forelimbs and sternum. Development 120:2065-2075.

Ye W, Shimamura K, Rubenstein JL, Hynes MA, Rosenthal A (1998) FGF and Shh signals control dopaminergic and serotonergic cell fate in the anterior neural plate. Cell 93:755-766.

Zetterström RH, Solomin L, Jansson L, Hoffer BJ, Olson L, Perlmann T (1997) Dopamine neuron agenesis in Nurr1-deficient mice. Science 276:248-250.

Zhou QY, Palmiter RD (1995) Dopamine-deficient mice are severely hypoactive, adipsic, and aphagic. Cell 83:1197-1209. 